\title{
Prevaccination Distribution of Human Papillomavirus Types in Italian Women with High-Risk Lesions and Cervical Neoplasia
}

\author{
Maria Antonia De Francesco ${ }^{a}$ Franco Gargiulo ${ }^{a}$ Claudio Schreiber $^{b}$ \\ Giuseppe Ciravolo $^{b}$ Federica Salinaro $^{b}$ Nino Manca $^{a}$ \\ a Institute of Microbiology and Virology, and bepartment of Obstetrics and Gynecology, Spedali Civili, \\ University of Brescia, Brescia, Italy
}

\section{Key Words}

Human papillomavirus $\cdot$ Cervical cancer

\begin{abstract}
Objectives: The aim of this study was to determine both human papillomavirus (HPV) prevalence and type distribution in cervical specimens of women with cytological abnormalities and to establish the association with high-grade lesions and cervical neoplasia in order to estimate the impact of an HPV vaccine in this region. Methods: Four hundred and ninety-three cervical specimens obtained from women undergoing routine cervical screening by liquid-based Pap smear were analyzed by Roche linear array HPV genotyping to identify HPV genotypes. Results: HPV 16 was the genotype detected most frequently, followed by HPV 31, 33 and 52. Multiple infections were frequent (58.5\%), but decreased with the increase of cervical severity. We found multiple infections composed by only LR types in 4 women: 3 had a histological diagnosis of cervical intraepithelial neoplasia (CIN) 3 and 1 a diagnosis of cervical cancer. HPV 16 alone was present in $24.6 \%$ of CIN 3 lesions and $40 \%$ of neoplasia. However, in our region, there are an additional $28 \%$ of cases of carcinoma in situ and $40 \%$ of cases of invasive cancer due to different HPV types that should be considered for eventual inclusion in second-generation HPV vaccines. Conclusions: These results highlight the importance of assessing individ-
\end{abstract}

ual types in the management and prediction of outcome of women with abnormal baseline cytology and may contribute to determine the potential efficacy of an HPV vaccine in clinical practice.

Copyright $\odot 2010$ S. Karger AG, Basel

\section{Introduction}

Invasive cervical cancer (ICC) is the second most common cancer among women worldwide, with an estimated incidence of 493,000 new cases and a mortality of 274,000 each year [1].

Persistent infection with high-risk human papillomavirus (HPV) types has been recognized as a necessary cause of cervical cancer $[2,3]$. Approximately $40 \mathrm{HPV}$ types are known to infect the anogenital tract, 15 of which $(16,18,31,33,35,39,45,51,52,56,58,59,68,73$ and 82$)$ have been implicated in the etiology of cervical cancer, and it is probable that another $3(26,53$ and 66$)$ are also involved [3]. In particular, HPV 16 and 18 are associated with $70-75 \%$ of invasive squamous cell cervical cancers and $80 \%$ of adenocarcinomas of the cervix $[4,5]$. HPV 16 and 18 are also associated with the development of other neoplasias of the genital tract such as vulvar and vaginal cancers and the preceding precancerous lesions [6].

\section{KARGER}

Fax +4161306 1234 E-Mail karger@karger.ch www.karger.com

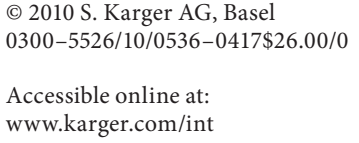


For this reason, currently available prophylactic vaccines contain only the two high-risk HPV genotypes [16, $18]$ identified as the most prevalent.

However, heterogeneity in HPV type distribution should be taken into account when predicting the effect of vaccines on the incidence of infection. HPV types other than 16 and 18 still account for 25-30\% of cervical cancer cases, and this percentage may be higher in certain populations. These geographic variations can play a crucial role in the effectiveness of an HPV vaccination and must be taken into consideration when designing policies against HPV.

We present below the results of a retrospective study performed in Brescia, Italy, to determine the prevalence of HPV infection for different grades of cervical neoplasia as determined by cytology and histology, as well as the overall age-specific prevalence of HPV infection in women with abnormal cytology. In addition, we assessed the type-specific HPV prevalence to estimate the preventive potential of HPV vaccines in preventing grade 2 or 3 squamous cervical intraepithelial neoplasia (CIN 2 or 3 ) or worse.

\section{Materials and Methods}

\section{Study Population}

This study includes 493 women aged 20-65 years (median 34.6) referred from primary care because abnormal cytology results according to TBS 1991 were found at routine gynecological examinations. An HPV test, another cytology and colposcopy were performed on all the patients. All patients provided informed consent for the tests.

\section{Colposcopy}

Colposcopic examination of the cervix was performed on all patients by several experienced colposcopists using an agreed protocol. Lesions in the transformation zone (TZ) were assessed by applying a $5 \%$ acetic acid and iodine solution under $8 \times 12 \mathrm{mag}$ nification. If colposcopy proved unsatisfactory, further exploration of the endocervix was carried out under $\times 20$ magnification using a Koogan speculum. International IFCPC nomenclature was used to classify the colposcopic pattern as either normal, abnormal TZ (ATZ), with minor changes suggesting low-grade CIN (CIN 1), ATZ with major changes suggesting CIN 2-3, or cancer.

\section{Biopsy and Histology}

Women with abnormal colposcopy results underwent punch biopsy. The pathological diagnosis of cervical lesions was performed according to a five-grade framework: CIN 1 (grade 1 cervical intraepithelial neoplasia, characterized by condilomatous lesions and/or light dysplasia); CIN 2 (moderate dysplasia); CIN 3 (severe dysplasia); carcinoma in situ (CIS); invasive carcinoma under Bethesda mode. Histological diagnoses were used as the reference standard.
Human Papillomavirus Testing

Sample Preparation

Cervical scrape specimens were collected into $20 \mathrm{ml}$ of PreservCyt LBC media (ThinPrepliquid PAP vial; Cytyc Corporation, Bedford, Mass., USA). For isolation of nucleic acid, $250 \mu \mathrm{l}$ of material were used with a Total Nucleic Acid Isolation Kit (Roche) as described by the manufacturer. Nucleic acid was resuspended in a final volume of $100 \mu \mathrm{l} ; 10 \mu \mathrm{l}$ was used for PCR analysis.

\section{PCR Amplification of HPV DNA}

The Linear Array HPV genotyping test (Roche Molecular Systems, Inc., Branchburg, N.J., USA) employs biotinylated primers to define a sequence of nucleotides within the polymorphic L1 region of the HPV genome, which is approximately $450 \mathrm{bp}$ long. A pool of HPV primers is used to amplify HPV DNA from 37 HPV genotypes, including 13 high-risk genotypes $(16,18,31,33$, $35,39,45,51,52,56,58,59$ and 68). An additional primer pair targets the human $\beta$-globin gene as a control for cell adequacy, extraction and amplification. Amplification was performed following the manufacturer's instructions. Following PCR amplification, hybridization reaction and detection were performed according to the manufacturer's instructions.

\section{Statistical Analysis}

Statistical analyses were performed using a $\chi^{2}$ test. The odds ratios, together with their $95 \%$ approximate confidence intervals were computed using the Mantel-Haenszel procedure. For all analyses, $\mathrm{p}<0.05$ was considered statistically significant.

\section{Results}

Cytological Diagnosis and HPV Prevalence according to Age

Among the 493 women in the study, 179 (36.3\%; 95\% CI $0.32-0.41$ ) had a cytological diagnosis of ASCUS, 213 (43.2\%; 95\% CI 0.38-0.48) had a cytological diagnosis of LSIL, 97 (19.6\%; 95\% CI 0.16-0.23) had a cytological diagnosis of HSIL, and 4 (0.8\%; 95\% CI 0-0.014) had a cytological diagnosis of cancer.

HPV DNA was detected in 426 of the 493 samples tested (86.3\%). Their eligibility for HPV testing was confirmed by positive $\beta$-globin results.

Overall, the results showed that HR HPV types alone were detected in 162 women (38\%; 95\% CI 0.33-0.42), while LR HPV types alone were detected in 81 women (19\%; 95\% CI 0.15-0.22). Single and multiple HPV infections were detected in 177 (41.5\%; 95\% CI 0.37-0.46) and 249 women (58.5\%; 95\% CI 0.53-0.62), respectively.

The mean age of the study population was 34.6 years (range 20-65). Ninety-one percent belonged to the target age group of 25-64 years, for whom screening is recommended [7]. Eight percent were younger than 25 and 1\% were older than 64 years. 


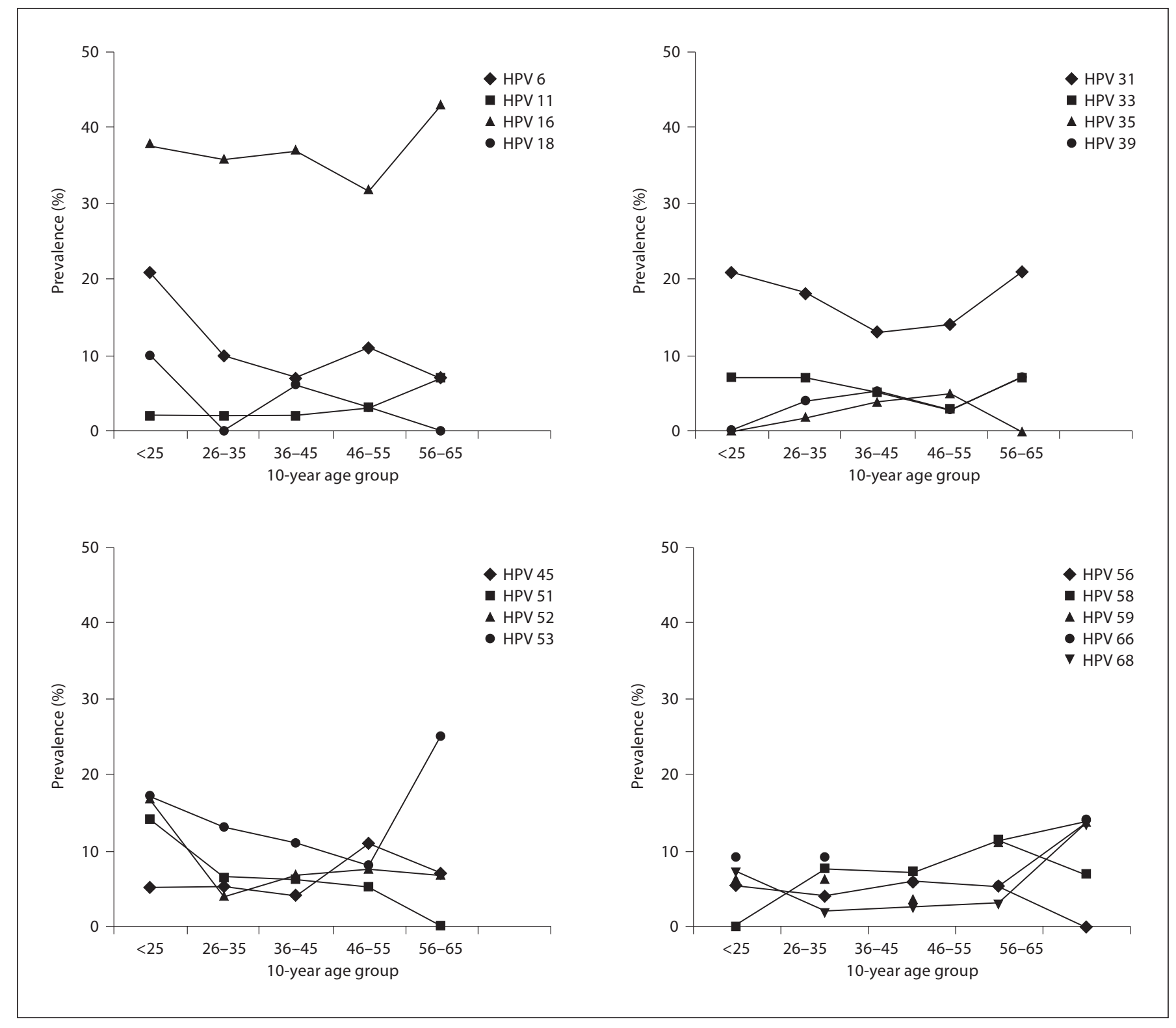

Fig. 1. Prevalence of cervical infections with HPV 6, HPV 11 and with individual HR HPV types, by age.

The prevalence of HR HPV infection was highest in the age group $<25$ years (range $7-38 \%$ ) and decreased progressively with increasing age (range $2-32 \%$ ) in the 46-55 years age group (fig. 1 ).

After that age, the HR HPV positivity rate varied, showing in most cases a second prevalence peak.

The HPV 6 prevalence peaked in the youngest age group ( $<25$ years), where $21 \%$ (95\% CI $0.1-0.33$ ) were positive, and then decreased with increasing age, while the HPV 11 prevalence was quite similar within the various age groups with a higher peak in older women (56-65 years).

\section{HPV Prevalence in Relation to Cytological and \\ Histological Diagnosis}

The most common HPV type detected in all the women was HPV 16, followed by HPV 31, 33, 52, 56 and 18.

HPV 16 was the most prevalent type independent of cytologic diagnosis (34\% of ASCUS, $33 \%$ of LSIL, $45 \%$ of HSIL and $66 \%$ of cancer samples) (table 1a). 
Table 1a. Prevalence of specific HPV types and group of types according to cytologic diagnosis

\begin{tabular}{|c|c|c|c|c|c|c|c|c|}
\hline \multirow[t]{2}{*}{ HPV type ${ }^{a}$} & \multicolumn{2}{|c|}{ ASCUS $(n=179)$} & \multicolumn{2}{|c|}{ LSIL $(n=213)$} & \multicolumn{2}{|c|}{ HSIL $(\mathrm{n}=97)$} & \multicolumn{2}{|c|}{ Cancer $(n=4)$} \\
\hline & $\%^{\mathrm{b}}$ & $95 \%$ CI & $\%$ & $95 \%$ CI & $\%$ & $95 \%$ CI & $\%$ & $95 \% \mathrm{CI}$ \\
\hline \multicolumn{9}{|l|}{ HR types } \\
\hline 16 & 34 & $0.27-0.41$ & 33 & $0.26-0.4$ & 45 & $0.34-0.56$ & 66 & $0.12-1.2$ \\
\hline 18 & 7.7 & $0.04-0.12$ & 3.8 & $0.01-0.07$ & 4.7 & $0-0.09$ & 0 & 0 \\
\hline 31 & 13.5 & $0.08-0.19$ & 18.4 & $0.13-0.24$ & 15.4 & $0.08-0.23$ & 0 & 0 \\
\hline 33 & 4.5 & $0.01-0.08$ & 5.4 & $0.02-0.09$ & 9.5 & $0.03-0.16$ & 0 & 0 \\
\hline 35 & 2.5 & $0-0.05$ & 3.8 & $0.01-0.07$ & 1.2 & $0-0.04$ & 0 & 0 \\
\hline 39 & 4.5 & $0.01-0.08$ & 3.8 & $0.01-0.07$ & 1.2 & $0-0.04$ & 0 & 0 \\
\hline 45 & 5.1 & $0.02-0.09$ & 8.3 & $0.04-0.12$ & 2.4 & $0-0.06$ & 0 & 0 \\
\hline 51 & 5.8 & $0.02-0.09$ & 8.3 & $0.04-0.12$ & 7.1 & $0.02-0.13$ & 0 & 0 \\
\hline 52 & 6.4 & $0.03-0.1$ & 4.3 & $0.01-0.07$ & 14.2 & $0.07-0.22$ & 0 & 0 \\
\hline 56 & 5.8 & $0.02-0.09$ & 5.9 & $0.02-0.09$ & 1.2 & $0-0.04$ & 0 & 0 \\
\hline 58 & 9 & $0.04-0.14$ & 6.5 & $0.03-0.1$ & 4.7 & $0-0.09$ & 0 & 0 \\
\hline 59 & 4.5 & $0.01-0.08$ & 8.7 & $0.05-0.13$ & 3.5 & $0-0.07$ & 0 & 0 \\
\hline 68 & 3.2 & $0-0.06$ & 3.8 & $0.01-0.07$ & 2.4 & $0-0.06$ & 0 & 0 \\
\hline \multicolumn{9}{|l|}{ Probable HR types } \\
\hline 53 & 14.8 & $0.09-0.2$ & 13 & $0.08-0.18$ & 10.7 & $0.04-0.17$ & 33 & $0-0.86$ \\
\hline 66 & 5.1 & $0.02-0.09$ & 11 & $0.06-0.16$ & 3.5 & $0-0.07$ & 33 & $0-0.86$ \\
\hline \multicolumn{9}{|l|}{ LR types } \\
\hline 6 & 19.3 & $0.13-0.26$ & 14 & $0.09-0.19$ & 1.2 & $0-0.04$ & 0 & 0 \\
\hline 11 & 3.8 & $0.01-0.07$ & 2.1 & $0-0.04$ & 0 & 0 & 0 & 0 \\
\hline 26 & 0 & 0 & 0.5 & $0-0.02$ & 0 & 0 & 0 & 0 \\
\hline 40 & 3.2 & $0-0.06$ & 2.7 & $0-0.05$ & 1.2 & $0-0.04$ & 0 & 0 \\
\hline 42 & 8.3 & $0.04-0.13$ & 10.8 & $0.06-0.15$ & 3.5 & $0-0.07$ & 0 & 0 \\
\hline 54 & 3.2 & $0-0.06$ & 8.7 & $0.05-0.13$ & 6 & $0.01-0.11$ & 0 & 0 \\
\hline 55 & 4.5 & $0.01-0.08$ & 3.2 & $0.01-0.06$ & 3.5 & $0-0.07$ & 0 & 0 \\
\hline 61 & 9 & $0.04-0.14$ & 13 & $0.08-0.18$ & 6 & $0.01-0.11$ & 33 & $0-0.86$ \\
\hline 62 & 9.6 & $0.05-0.14$ & 9.7 & $0.05-0.14$ & 3.5 & $0-0.07$ & 0 & 0 \\
\hline 64 & 0 & 0 & 0 & 0 & 0 & 0 & 0 & 0 \\
\hline 67 & 1.9 & $0-0.04$ & 2.7 & $0-0.05$ & 2.4 & $0-0.06$ & 0 & 0 \\
\hline 69 & 0 & 0 & 0 & 0 & 0 & 0 & 0 & 0 \\
\hline 70 & 2.5 & $0-0.05$ & 2.1 & $0-0.04$ & 2.4 & $0-0.06$ & 0 & 0 \\
\hline 71 & 0.6 & $0.01-0.02$ & 0.5 & $0-0.02$ & 0 & 0 & 0 & 0 \\
\hline 72 & 0 & 0 & 1.6 & $0-0.03$ & 0 & 0 & 0 & 0 \\
\hline 73 & 6.4 & $0.03-0.1$ & 4.9 & $0.02-0.08$ & 6 & $0.01-0.11$ & 0 & 0 \\
\hline 81 & 6.4 & $0.03-0.1$ & 3.8 & $0.01-0.07$ & 2.4 & $0-0.06$ & 0 & 0 \\
\hline 82 & 0 & 0 & 0 & 0 & 0 & 0 & 0 & 0 \\
\hline 83 & 0.6 & $0-0.02$ & 3.8 & $0.01-0.07$ & 3.5 & $0-0.07$ & 0 & 0 \\
\hline 84 & 7 & $0.03-0.11$ & 6.5 & $0.03-0.1$ & 2.4 & $0-0.06$ & 0 & 0 \\
\hline IS39 & 0 & 0 & 0 & 0 & 0 & 0 & 0 & 0 \\
\hline CP6108 & 5.8 & $0.02-0.09$ & 8.1 & $0.04-0.12$ & 7.1 & $0.02-0.13$ & 0 & 0 \\
\hline \multicolumn{9}{|l|}{ Groups of types } \\
\hline $6 / 11$ & 23.2 & $0.17-0.3$ & 8.1 & $0.04-0.12$ & 1.2 & $0-0.04$ & 0 & 0 \\
\hline $16 / 18$ & 40.6 & $0.33-0.48$ & 36.4 & $0.29-0.43$ & 50 & $0.39-0.61$ & 66 & 0.12 \\
\hline 6/11/16/18 & 59 & $0.51-0.67$ & 41 & $0.34-0.48$ & 51 & $0.40-0.62$ & 66 & 0.12 \\
\hline $6 / 11 / 16 / 18 / 31$ & 66 & $0.59-0.73$ & 56 & $0.49-0.63$ & 63 & $0.53-0.73$ & 66 & 0.12 \\
\hline 6/11/16/18/31/45 & 69 & $0.62-0.76$ & 59.2 & $0.52-0.66$ & 64.2 & $0.54-0.74$ & 66 & 0.12 \\
\hline
\end{tabular}

HPV 31 was common in both LSIL and HSIL lesions (18.4 and 15.4\%, respectively). As the severity of lesions increased, the percentage of samples containing HPV 33 and 51 also tended to increase. The frequency of HPV 18 in HSIL was similar to that of HPV $58(4.7 \%)$ and was inferior to the frequency of HPV 51 (7.1\%). The most com- mon LR type found in ASCUS and LSIL was HPV 6 (19.3 and 14\%, respectively); while the LR types more frequently associated with all grades of cytology were CP6108, HPV 54 and 61. Finally, HPV 11 was present in $3.8 \%$ of women with ASCUS, $2.1 \%$ of women with LSIL and in none of the women with HSIL or cancer diagnosis. 
Table 1b. Prevalence of specific HPV types and group of types according to histologic diagnosis

\begin{tabular}{|c|c|c|c|c|c|c|c|c|c|c|}
\hline \multirow[t]{2}{*}{ HPV type ${ }^{a}$} & \multicolumn{2}{|c|}{ CIN $1(n=179)$} & \multicolumn{2}{|c|}{ CIN $2(n=133)$} & \multicolumn{2}{|c|}{ CIN $3(n=77)$} & \multicolumn{2}{|c|}{ CIS $(n=32)$} & \multicolumn{2}{|c|}{$\mathrm{IC}(\mathrm{n}=5)$} \\
\hline & $\%^{\mathrm{b}}$ & $95 \%$ CI & $\%$ & $95 \%$ CI & $\%$ & $95 \% \mathrm{CI}$ & $\%$ & $95 \%$ CI & $\%$ & $95 \%$ CI \\
\hline \multicolumn{11}{|l|}{ HR types } \\
\hline 16 & 19.5 & $0.14-0.25$ & 43.6 & $0.35-0.52$ & 49.3 & $0.38-0.60$ & 65.6 & $0.49-0.82$ & 60 & $0.17-1.03$ \\
\hline 18 & 7.2 & $0.03-0.11$ & 3 & $0-0.06$ & 5.2 & $0-0.1$ & 6.2 & $0-0.15$ & 0 & 0 \\
\hline 31 & 13.4 & $0.08-0.18$ & 18.7 & $0.12-0.25$ & 19.4 & $0.11-0.28$ & 15.6 & $0.03-0.28$ & 0 & 0 \\
\hline 33 & 3.3 & $0.01-0.06$ & 6 & $0.02-0.1$ & 9.1 & $0.03-0.16$ & 12.5 & $0.01-0.24$ & 0 & 0 \\
\hline 35 & 2.2 & $0-0.04$ & 5.2 & $0.01-0.09$ & 0 & 0 & 3.1 & $0-0.09$ & 0 & 0 \\
\hline 39 & 4 & $0.01-0.07$ & 4.5 & $0.01-0.08$ & 1.3 & $0-0.04$ & 0 & 0 & 0 & 0 \\
\hline 45 & 6.1 & $0.03-0.1$ & 6 & $0.02-0.1$ & 3.9 & $0-0.08$ & 0 & 0 & 20 & $0-0.55$ \\
\hline 51 & 6.1 & $0.03-0.1$ & 9 & $0.04-0.14$ & 3.9 & $0-0.08$ & 3.1 & $0-0.09$ & 20 & $0-0.55$ \\
\hline 52 & 6.1 & $0.03-0.1$ & 6.7 & $0.02-0.11$ & 10.3 & $0.04-0.17$ & 9.3 & $0-0.19$ & 0 & 0 \\
\hline 56 & 4.4 & $0.01-0.07$ & 7.5 & $0.03-0.12$ & 1.3 & $0-0.04$ & 6.2 & $0-0.15$ & 0 & 0 \\
\hline 58 & 6.1 & $0.03-0.1$ & 6 & $0.02-0.1$ & 12.9 & $0.05-0.2$ & 3.1 & $0-0.09$ & 0 & 0 \\
\hline 59 & 7.8 & $0.04-0.12$ & 5.2 & $0.01-0.09$ & 5.2 & $0-0.1$ & 0 & 0 & 20 & $0-0.55$ \\
\hline 68 & 5 & $0.02-0.08$ & 3 & $0-0.06$ & 0 & 0 & 0 & 0 & 0 & 0 \\
\hline \multicolumn{11}{|l|}{ Probable HR types } \\
\hline 53 & 14 & $0.09-0.19$ & 16.5 & $0.1-0.23$ & 11.6 & $0.04-0.19$ & 6.2 & $0-0.15$ & 0 & 0 \\
\hline 66 & 9 & $0.05-0.13$ & 9 & $0.04-0.14$ & 5.2 & $0-0.1$ & 3.1 & $0-0.09$ & 0 & 0 \\
\hline \multicolumn{11}{|l|}{ LR types } \\
\hline 6 & 20.1 & $0.14-0.26$ & 3.7 & $0-0.07$ & 2.6 & $0-0.06$ & 0 & 0 & 0 & 0 \\
\hline 11 & 4 & $0.01-0.07$ & 2.2 & $0-0.05$ & 0 & 0 & 0 & 0 & 0 & 0 \\
\hline 26 & 0.5 & $0-0.02$ & 0 & 0 & 0 & 0 & 0 & 0 & 0 & 0 \\
\hline 40 & 4 & $0.01-0.07$ & 1.5 & $0-0.04$ & 1.3 & $0-0.04$ & 3.1 & $0-0.09$ & 0 & 0 \\
\hline 42 & 12.8 & $0.08-0.18$ & 6.7 & $0.02-0.11$ & 2.6 & $0-0.06$ & 3.1 & $0-0.09$ & 20 & $0-0.55$ \\
\hline 54 & 6.7 & $0.03-0.1$ & 6.7 & $0.02-0.11$ & 6.5 & $0.01-0.12$ & 0 & 0 & 0 & 0 \\
\hline 55 & 5.5 & $0.02-0.09$ & 2.2 & $0-0.05$ & 3.9 & $0-0.08$ & 0 & 0 & 0 & 0 \\
\hline 61 & 11 & $0.06-0.16$ & 10.5 & $0.05-0.16$ & 10.3 & $0.04-0.17$ & 6.2 & $0-0.15$ & 20 & $0-0.55$ \\
\hline 62 & 14 & $0.09-0.19$ & 6 & $0.02-0.1$ & 2.6 & $0-0.06$ & 3.1 & $0-0.09$ & 0 & 0 \\
\hline 64 & 0 & 0 & 0 & 0 & 0 & 0 & 0 & 0 & 0 & 0 \\
\hline 67 & 1.6 & $0-0.03$ & 2.2 & $0-0.05$ & 2.6 & $0-0.06$ & 0 & 0 & 20 & $0-0.55$ \\
\hline 69 & 0 & 0 & 0 & 0 & 0 & 0 & 0 & 0 & 0 & 0 \\
\hline 70 & 2.8 & $0-0.05$ & 2.2 & $0-0.05$ & 2.6 & $0-0.6$ & 0 & 0 & 0 & 0 \\
\hline 71 & 0.5 & $0-0.02$ & 0 & 0 & 1.3 & $0-0.04$ & 0 & 0 & 0 & 0 \\
\hline 72 & 1.6 & $0-0.03$ & 0 & 0 & 0 & 0 & 0 & 0 & 0 & 0 \\
\hline 73 & 7.8 & $0.04-0.12$ & 3 & $0-0.06$ & 5.2 & $0-0.1$ & 0 & 0 & 20 & $0-0.55$ \\
\hline 81 & 4.4 & $0.01-0.07$ & 4.5 & $0.01-0.08$ & 1.3 & $0-0.04$ & 0 & 0 & 0 & 0 \\
\hline 82 & 0 & 0 & 0 & 0 & 0 & 0 & 0 & 0 & 0 & 0 \\
\hline 83 & 2.8 & $0-0.05$ & 3 & $0-0.06$ & 0 & 0 & 3.1 & $0-0.09$ & 0 & 0 \\
\hline 84 & 7.2 & $0.03-0.11$ & 8.2 & $0.04-0.13$ & 1.3 & $0-0.04$ & 0 & 0 & 0 & 0 \\
\hline IS39 & 0 & 0 & 0 & 0 & 0 & 0 & 0 & 0 & 0 & 0 \\
\hline CP6108 & 9.4 & $0.05-0.14$ & 8.2 & $0.04-0.13$ & 2.6 & $0-0.06$ & 0 & 0 & 0 & 0 \\
\hline \multicolumn{11}{|l|}{ Groups of types } \\
\hline $6 / 11$ & 24 & $0.18-0.3$ & 5.2 & $0.01-0.09$ & 2.6 & $0-0.06$ & 0 & 0 & 0 & 0 \\
\hline $16 / 18$ & 26.8 & $0.2-0.33$ & 45.8 & $0.37-0.54$ & 52 & $0.41-0.63$ & 71.8 & $0.56-0.87$ & 60 & $0.17-1.03$ \\
\hline $6 / 11 / 16 / 18$ & 44.6 & $0.37-0.52$ & 48.8 & $0.40-0.57$ & 53.2 & $0.42-0.64$ & 71.8 & $0.56-0.87$ & 60 & $0.17-1.03$ \\
\hline $6 / 11 / 16 / 18 / 31$ & 52.5 & $0.45-0.6$ & 65.4 & $0.57-0.73$ & 68.8 & $0.58-0.79$ & 87.5 & $0.76-0.99$ & 60 & $0.17-1.03$ \\
\hline $6 / 11 / 16 / 18 / 31 / 45$ & 57.5 & $0.50-0.65$ & 68.4 & $0.60-0.76$ & 72.7 & $0.63-0.83$ & 87.5 & $0.76-0.99$ & 60 & $0.17-1.03$ \\
\hline
\end{tabular}

CIS = Carcinoma in situ; IC = invasive cancer. ${ }^{a}$ HPV types as they appear on the nylon HPV strips. ${ }^{\mathrm{b}}$ Number of specimens containing the designated HPV type (as a single HPV type or in multiple infections). The most prevalent HPV types identified within each group are highlighted in bold type.

In women with CIN 1 or CIN 2 HPV 16, 31, 51, 52 and 56 were the predominant types (table $1 \mathrm{~b}$ ). However, in samples from women with CIN 3, CIS and IC (invasive cancer) HPV 16 was by far the most prevalent HPV type (49.3, 65.6 and $60 \%$, respectively) compared with the other common HR HPV types, i.e. HPV 31 (19.4, 15.6\% and none, respectively), HPV 33 (9.1, 12.5\% and none, respectively), and HPV 52 (10.3, 9.3\% and none, respectively). HPV 18 and HPV 56 had the same prevalence in CIS (6.2\%), while HPV 45 was less common (only $1.3 \%$ in CIN 3 and none in CIS). Comparing the two probable high-risk HPV (53 and 66), we found a higher prevalence 

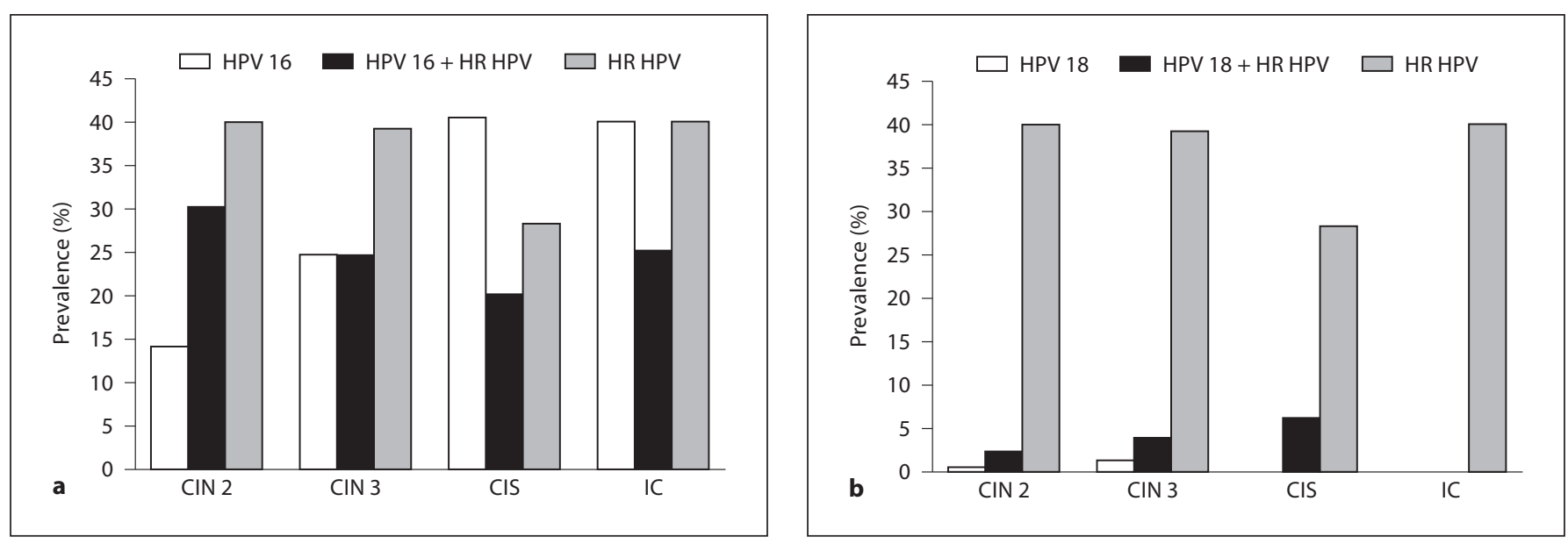

Fig. 2. Prevalence (\%) of HPV 16 alone (a) or HPV 18 alone (b), HPV 16 (a) or HPV 18 (b) with other high-risk HPV types and other high-risk HPV types (without HPV 16 or 18) in CIN 2, CIN 3, CIS and in IC histological diagnosis.

of HPV 53 (11.6\% in CIN 3 and 6.2\% in CIS) and a moderate prevalence of HPV 66 (5.2\% in CIN 3 and $3.1 \%$ in CIS). Among the LR HPV types, HPV 6 was the most frequent type in CIN 1, while the LR HPV types associated with all grades of histological diagnosis were HPV 42 (12.8\% in CIN 1, 6.7\% in CIN 2, 2.6\% in CIN 3, 3.1\% in CIS and 20\% in invasive cancer) and HPV 61 (11\% in CIN 1, 10.5\% in CIN 2, 10.3 in CIN 3, 6.2\% in CIS and $20 \%$ in invasive cancer). Most of these LR types were present together with other HR HPV types. However, we found multiple infections by only LR types in 3 samples from women with a histological diagnosis of CIN 3 and in 1 sample from a woman with a diagnosis of invasive cancer. In this population, we did not find any HPV 64, 69,82 and IS39.

The occurrence of HPV 16 and HPV 18 alone, respectively, of HPV 16 and HPV 18 together with other HR HPV types and of other HR HPV types alone without HPV 16 or 18 in CIN 2+ and >CIN 2 lesions is shown in figure $2 \mathrm{a}, 2 \mathrm{~b}$. The prevalence of HPV 16 alone increased from $14 \%$ in CIN 2 to $40 \%$ in CIS and IC, the prevalence of HPV 18 alone ranged from $0.7 \%$ in CIN 2 to $1.3 \%$ in CIN 3 while it was absent in CIS and IC cases. The simultaneous presence of HPV 16 and other HR types was found more frequently than HPV 16 alone only in CIN 2 (30\%), while it decreased in CIS (20\%) and IC (25\%). On the contrary, for HPV 18, occurrence of the simultaneous presence of other HR HPV increased from $2.3 \%$ in CIN 2 to $6.3 \%$ in CIS. Otherwise, the occurrence of HR HPV alone was higher than HPV 16 alone in CIN 2 and CIN 3 (about 39\%), lower in CIS (28.1\%) and the same in IC (40\%).

\section{Risk of High-Grade Lesions and Cervical Neoplasia according to Specific HPV Prevalence}

To evaluate the net effect of multiple infections on high-grade CIN (CIN 2-CIN 3)/cancer (CIS, IC), we used CIN 1 as reference category (table 2). In this model, we found that the prevalence of high-grade $\mathrm{CIN} /$ cancer was significantly higher in women with a single infection than in women with increasing number of HPV infection $(\mathrm{p}<0.05)$. In addition, the OR of high-grade CIN/cancer among women with single as compared with multiple HPV infection was $1.9(\mathrm{CI}=1.2-2.99)$.

The associations between different grades of histological abnormalities and different combinations of HPV 16 and other HR HPV types are shown in table 3. Compared with women who were negative for HPV 16, women infected with other HR HPV types had an increased risk of CIN 2, CIN 3 or worse. For this reason, we calculated the odds ratios of these lesions according to HR HPV types other than HPV 16 for infections by single type as compared to the remaining study sample (table 4). Due to the small number of single infections, ORs could not be calculated for some strains.

The results showed that the likelihood of CIN 2 was higher among women infected with most HR HPV types than in those infected with HPV 16, while in high-grade lesions (CIN 3 or worse) we observed a maximal oncogenic risk associated with HPV 16 infections. 
Table 2. Association between histological outcomes and number of infecting HPV types

\begin{tabular}{lccccccr}
\hline Histology & \multicolumn{2}{l}{ Number of infecting HPV types } & & \\
\cline { 2 - 8 } & 1 & 2 & 3 & 4 & 5 & $>5$ & total \\
\hline CIN 1 & $65(36.7)$ & $51(41.4)$ & $31(50.8)$ & $16(45.7)$ & $7(46.6)$ & $9(60)$ & 179 \\
CIN 2 & $52(29.3)$ & $40(32.5)$ & $19(31.1)$ & $11(31.4)$ & $7(46.6)$ & $4(26.6)$ & 133 \\
CIN 3 & $38(21.4)$ & $23(18.6)$ & $10(16.3)$ & $4(11.4)$ & 00 & $2(13.3)$ & 77 \\
CIS & $20(11.2)$ & $8(6.5)$ & 00 & $3(8.5)$ & $1(6.6)$ & 00 & 32 \\
IC & $2(1.1)$ & $1(0.81)$ & $1(1.6)$ & $1(2.8)$ & 00 & 00 & 5 \\
\hline Total & 177 & 123 & 61 & 35 & 15 & 15 & 426 \\
\hline
\end{tabular}

CIS $=$ Carcinoma in situ; IC = invasive cancer. Figures shown in parentheses are percentages.

\section{Discussion}

The human papillomavirus has a global distribution [8]. The prevalence of the different types of HPV worldwide has implications for the effectiveness of HPV vaccination against HPV-induced carcinogenesis. It has been suggested that a worldwide vaccine against HPV 16 and HPV 18 may prevent a larger proportion of cervical carcinoma cases in Europe, North America and Australia than in Africa, South America and Asia $[8,9]$. HPV 16 represents the most commonly identified HPV type in low- and high-grade lesions as well as cervical cancer worldwide $[8,9]$. A recent meta-analysis by Clifford et al. [10] showed that in high-grade lesions (HSIL) and in ICC HPV 16 was the most common, and HPV 18 the second most common type in all continents. Combined HPV $16 / 18$ prevalence among ICC cases was slightly higher in Europe, North America and Australia (74-77\%) than in Africa, Asia and South/Central America (65-70\%). In our results too, the most common HPV type in all cytology samples and high-grade histology samples was HPV 16 , which is in agreement with other studies $[11,13]$. Although significantly less frequent than HPV 16, both HPV 31 and 52 were also common in this study, in accordance with our previous results [14]. HPV 31 has also been found to be widespread in Europe [12, 15]. Our results revealed that HPV 16 was present in $14 \%$ of samples from women with CIN 2 lesions and in $24.6 \%$ of women with CIN 3 lesions, while HPV 18 was present in less than $1 \%$ of women with CIN 2 lesions and in $1.3 \%$ of women with CIN 3 lesions. At this point, if we assume a $100 \%$ efficacy and coverage of an HPV 16/18 vaccine, our results suggest that the vaccine could prevent at least $26 \%$ of all CIN 3 lesions. As HPV 16 and HPV 18 are the caus-
Table 3. Odds ratios and 95\% CIs of histological results according to the presence of HPV 16 alone or of HR HPV types alone or of a combination of both

\begin{tabular}{llcc}
\hline Histology & Variable & OR & $95 \%$ CI \\
\hline CIN 2 & & & \\
& HPV 16 & 0.84 & $0.44-1.56$ \\
& HPV 16 + HR HPV types & 5 & $2.7-9.2$ \\
& HR HPV types & 20.9 & $9.4-47.6$ \\
$\geq$ CIN 3 & & 3.99 & \\
& HPV 16 & 2.5 & $1.4-7.1$ \\
& HPV 16 + HR HPV types & 7.7 & $4.1-14.5$ \\
& HR HPV types &
\end{tabular}

al agents even in the presence of other HR HPV types, the preventive potential could be $53.1 \%$. In this scenario, however, our data indicate that $39 \%$ of CIN 2 and CIN 3 lesions are entirely sustained by HPV types that are not targeted by currently available multivalent vaccine. For this reason, we evaluated the ORs for CIN 2 and $\geq \mathrm{CIN}$ 3 for infection with HPV 16 alone or with other HR types. Our data showed that the risk of CIN 2 or $\geq \mathrm{CIN}$ 3 was much higher in women with HR HPV infection other than HPV 16 alone or in association with other high-risk HPV types. When the ORs for CIN 2 and $\geq$ CIN 3 were related to a single HR HPV type infection, we found that women infected with HR HPV types, except for HPV 51, had a higher risk of CIN 2 lesions than women infected with HPV 16 alone. On the contrary, the maximal oncogenic risk of $\geq \mathrm{CIN} 3$ lesions was associated with infection with HPV 16 alone, even if other HR HPV types had a high risk such as HPV 31, 33 and 52. 
Table 4. Odds ratios and 95\% CIs of CIN 2 and $\geq \mathrm{CIN} 3$ attributable to infection with a given HR HPV type

\begin{tabular}{|c|c|c|c|c|}
\hline \multirow[t]{2}{*}{ HPV type } & \multicolumn{2}{|l|}{ CIN 2} & \multicolumn{2}{|l|}{$\geq \mathrm{CIN} 3$} \\
\hline & OR & $95 \% \mathrm{CI}$ & OR & $95 \% \mathrm{CI}$ \\
\hline 18 & 1.1 & $0.04-15.5$ & $1.37(0.04-19.4)$ & $1.24(0.06-3.31)$ \\
\hline 31 & 0.92 & $0.36-2.30$ & $2.7(1.16-6.43)$ & $1.9(1.11-2.79)$ \\
\hline 33 & 0.87 & $0.11-5.17$ & $2.1(0.36-11.1)$ & $1.6(0.44-3)$ \\
\hline 35 & undefined & undefined & undefined & undefined \\
\hline 39 & 2.2 & $0.06-8.1$ & undefined & undefined \\
\hline 45 & 2.2 & $0.22-22.3$ & $0.91(0.03-9.91)$ & $0.93(0.05-2.96)$ \\
\hline 51 & 0.54 & $0.02-5.23$ & undefined & undefined \\
\hline 52 & undefined & undefined & $2.8(0.44-17.5)$ & $1.9(0.52-3.31)$ \\
\hline 56 & 3.3 & $0.45-29$ & undefined & undefined \\
\hline 58 & $1.3(0.24-6.48)$ & $1.2(0.32-2.43)$ & $1.6(0.3-8.11)$ & $1.4(0.38-2.85)$ \\
\hline 59 & $1.1(0.04-15.5)$ & $1(0.05-2.83)$ & undefined & undefined \\
\hline 53 & $1.7(0.29-8.95)$ & $1.4(0.37-2.61)$ & undefined & undefined \\
\hline
\end{tabular}

Up to this point, the incremental risk observed for HR HPV types was probably due to infections sustained by different high-risk strains. These observations support the notion that any additional infecting virus is contributory to the risk of CIN and no single HPV type exerts a maximal oncogenic effect. In agreement with other studies [16-18], we found that HPV prevalence had high rates in young women, then there was a decrease in middle-aged groups followed by a second rise in postmenopausal age groups. This second rise might be related to sexual behavior or to a reactivation of a latent HPV infection, even if this last hypothesis remains difficult to prove.

We found that infection with multiple HPV types was very frequent (58.5\%). This high frequency of HPV coinfections was in accordance with our previous study [14] and was entirely consistent with that reported by other international studies $[11,19,20]$. As already observed [14], also in this study the proportion of multiple type infections among HPV-positive patients decreased in highgrade lesions and cancer compared with low-grade lesions. This corresponds with the multi-stage cancer model, by which one HPV type tends to predominate increasingly with the increase of cervical lesion severity [21].

In fact, we found a 1.9 times higher risk of high-grade $\mathrm{CIN} /$ cancer in women with a single infection than women affected by more than one HPV type.

However, in terms of risk to develop high-grade lesions, the clinical importance of finding multiple carcinogenic types in a specimen is still under debate $[19,20]$. Whenever multiple HPV types are identified in a clini- cal specimen with one or more histologically confirmed lesions (i.e. coexistence of CIN 1 and CIN 3), causal allocation of individual viral types and lesions remains elusive and biomarkers are still under development. Direct virus-virus interaction on the rate of clearance are not strong if present. It is not known whether, following HPV vaccination, the lower prevalence of HPV 16 and HPV 18 would affect the carcinogenicity of currently less important types. This is particularly important in the light of our finding that we had four multiple infections composed exclusively of HPV types that are not recognized in the category of high risk types. These infections were present in three high-grade dysplasias and one invasive cancer. The composition of these multiple infections was extremely varied, comprising two probable high-risk types such as HPV 53 and 66, and HPV 73 included in the high-risk class by a wide epidemiologic study [22]. This study has some limitations: the analysis of a selected group of patients, not representative of the general population, and the cross-sectional design of the study. Extension of the cohort and followup of HPV-positive women will provide more relevant data about the cumulative incidence of CIN 2, CIN 3 and cancer associated with type-specific HPV infections.

However, with the current study, an important step has been made for a comprehensive series of surveillance activities regarding HPV vaccination. Removing the most oncogenic HPV types through vaccination will probably result in fewer cervical abnormalities. Nevertheless, this decrease will be smaller than usually expected because a multitude of HPV types cause cervical in- 
traepithelial neoplasia. This should not be considered as vaccine failure because HPV 16 and, to a lesser degree, HPV 18 are particularly carcinogenic and their elimination would therefore preferentially prevent progressive lesions.
Another unresolved issue is the prognostic value of multiple infections for high-grade lesions and cervical cancer. Perhaps persistence of a type over time, analyzed by sensitive type-specific in situ hybridization or by use of mRNA-based tests, may clarify the natural history of HPV infections at the type-specific level.

\section{References}

1 Ferlay J, Bray F, Pisani P, Parkin DM: GLOBOCAN 2002: Cancer Incidence, Mortality, and Prevalence Worldwide, IARC Cancer Base No 5 Version 2.0. Lyon, IARC Press, 2004.

-2 Walboomers JMM, Jacob MV, Manos MM, et al: Human papillomavirus is a necessary cause of invasive cervical cancer worldwide. J Pathol 1999;189:12-19.

3 Munoz N, Bosch FX, de Sanjos S, et al: Epidemiologic classification of human papillomavirus types associated with cervical cancer. N Engl J Med 2003;384:518-527.

4 Smith JS, Lindsay L, Hoots B, et al: Human papillomavirus type distribution in invasive cervical cancer and high grade cervical lesions: a meta-analysis update. Int J Cancer 2007;121:621-632.

-5 Castellsague X, Diaz M, de Sanjose S, et al: Worldwide human papillomavirus etiology of cervical adenocarcinoma and its cofactors: implications for screening and prevention. J Natl Cancer Inst 2006;98:303-315.

-6 Munoz N, Castellsague X, Berrington de Gonzales A, et al: HPV in the etiology of human cancer. Vaccine Suppl 3 2006;24:1-10.

7 Barzon L, Giorgi C, Buonaguro FM, et al: Guidelines of the Italian Society for Virology on HPV testing and vaccination for cervical cancer prevention. Infect Agents Cancer 2008;3:14.

8 Clifford GM, Smith JS, Plummer M, et al: Human papillomavirus types in invasive cervical cancer worldwide: a meta-analysis. Br J Cancer 2003;88:63-73.
9 Clifford GM, Smith JS, Aguardo T, et al: Comparison of HPV type distribution in high grade cervical lesions and cervical cancer: a meta-analysis. Br J Cancer 2003;89: 101-105.

10 Smith JS, Lindsay L, Hoots B, et al: Human papillomavirus type distribution in invasive cervical cancer and high-grade cervical lesions: a meta-analysis update. Int J Cancer 2007;121:621-632.

11 Herrero R, Castle P, Schiffman M, et al: Epidemiologic profile of type-specific human papillomavirus infection and cervical neoplasia in Guanacaste, Costa Rica. J Infect Dis 2005;191:1796-1807.

12 Clifford GM, Gallus S, Herrero R, et al: Worldwide distribution of human papillomavirus types in cytologically normal women in the International Agency for Research on Cancer HPV prevalence surveys: a pooled analysis. Lancet 2005;366:991-998.

13 Dal Bello B, Spinillo A, Alberizzi P, et al: Cervical infections by multiple human papillomavirus (HPV) genotypes: prevalence and impact on the risk of precancerous epithelial lesions. J Med Virol 2009;81:703-712.

14 Gargiulo F, De Francesco MA, Schreiber C et al: Prevalence and distribution of single and multiple HPV infections in cytologically abnormal cervical samples from Italian women. Vir Res 2007;125:176-182.

15 Clifford GM, Rana RK, Franceschi S, et al: Pimenta, human papillomavirus genotype distribution in low-grade cervical lesions: comparison by geographic region and with cervical cancer. Cancer Epidemiol Biomarkers Prev 2005;14:1157-1164.
16 Kitchener HC, Almonte M, Wheeler P, et al: $\mathrm{HPV}$ testing in routine cervical screening: cross sectional data from the ARTISTIC trial. Br J Cancer 2006;95:56-61.

17 Franceschi S, Herrero R, Clifford GM, et al: Variations in the age-specific curves of human papillomavirus prevalence worldwide. Int J Cancer 2006;119:2677-2684.

18 Bosch FX, Burchell AN, Schiffman M, et al: Epidemiology and natural history of human papillomavirus infections and type-specific implications in cervical neoplasia. Vaccine 2008;26(suppl):1-16.

19 Trottier H, Mahmud S, Costa MC, et al: Human papillomavirus infections with multiple types and risk of cervical neoplasia. Cancer Epidemiol Biomarkers Prev 2006; 15: 1274-1280.

20 Wheeler CM, Hunt WC, Schiffman M, et al: Human papillomavirus genotypes and the cumulative 2-year risk of cervical precancer. J Infect Dis 2006;194:1291-1299.

-21 Schiffman M, Herrero R, DeSalle R, et al: The carcinogenicity of human papillomavirus types reflects viral evolution. Virology 2005;337:76-84.

22 Munoz N, Bosch F, de Sanjose S, et al: Epidemiologic classification of human papillomavirus types associated with cervical cancer. N Engl J Med 2003;348:518-527. 